\title{
CD56+ immune cell infiltration and MICA are decreased in breast lobules with fibrocystic changes
}

\author{
Daniel Kerekes $^{1} \cdot$ Daniel W. Visscher $^{2} \cdot$ Tanya L. Hoskin $^{3} \cdot$ Derek C. Radisky ${ }^{4} \cdot$ Rushin D. Brahmbhatt $^{1}$. \\ Alvaro Pena $^{1} \cdot$ Marlene H. Frost $^{5} \cdot$ Muhammad Arshad $^{1} \cdot$ Melody Stallings-Mann $^{4} \cdot$ Stacey J. Winham ${ }^{3}$. \\ Linda Murphy ${ }^{5}$ Lori Denison ${ }^{6}$ Jodi M. Carter ${ }^{2} \cdot$ Keith L. Knutson $^{7} \cdot$ Amy C. Degnim $^{1}$
}

Received: 14 July 2017 / Accepted: 27 October 2017 / Published online: 1 November 2017

(c) The Author(s) 2017. This article is an open access publication

\begin{abstract}
Purpose While the role of natural killer (NK) cells in breast cancer therapy has been investigated, little information is known about NK cell function and presence in nonmalignant and premalignant breast tissue. Here, we investigate and quantify NK cell marker CD56 and activating ligand MICA in breast tissue with benign breast disease.

Methods Serial tissue sections from 88 subjects, 44 with benign breast disease (BBD) who remained cancer-free, and 44 with BBD who later developed cancer, were stained with $\mathrm{H} \& \mathrm{E}$, anti-MICA, and anti-CD56. Up to ten representative lobules were identified on each section. Using digital image analysis, MICA and CD56 densities were determined for each lobule, reported as percent of pixels in the lobule that registered as stained by each antibody. Analyses were performed on a per-subject and per-lobule basis.
\end{abstract}

Electronic supplementary material The online version of this article (https://doi.org/10.1007/s10549-017-4558-0) contains supplementary material, which is available to authorized users.

Amy C. Degnim

degnim.amy@mayo.edu

1 Department of Surgery, Mayo Clinic, 200 First Street SW, Rochester, MN 55905, USA

2 Division of Anatomic Pathology, Mayo Clinic, Rochester, MN, USA

3 Division of Biomedical Statistics and Informatics, Mayo Clinic, Rochester, MN, USA

4 Cancer Biology, Mayo Clinic, Jacksonville, FL, USA

5 Women's Cancer Program, Mayo Clinic, Rochester, MN, USA

6 Information Technology, Mayo Clinic, Rochester, MN, USA

7 Immunology, Mayo Clinic, Jacksonville, FL, USA
Results Per-subject multivariate analyses showed associations of CD56 and MICA with age: CD56 was increased in older subjects $(p=0.03)$, while MICA was increased in younger subjects $(p=0.005)$. Per-lobule analyses showed that CD56 and MICA levels were both decreased in lobules with fibrocystic change, with median levels of CD56 and MICA staining, respectively, at 0.31 and $7.0 \%$ in fibrocystic lobules compared to 0.76 and $12.2 \%$ in lobules without fibrocystic change ( $p<0.001$ for each). Among fibrocystic lobules, proliferative/atypical lobules showed significantly lower expression compared to nonproliferative lobules for MICA $(p=0.02)$ but not for CD56 $(p=0.80)$.

Conclusion Levels of CD56+ NK cells and activating ligand MICA were decreased in breast lobules with fibrocystic change, and MICA levels showed a significant stepwise decrease with increasing histopathologic abnormality. MICA levels were also significantly decreased in older subjects, who generally have higher risk of developing cancer. These findings advance a model in which MICA promotes cytotoxic activity in CD56+ NK cells to protect against tumorigenesis in breast lobules, and suggest further research is warranted.

Keywords Natural killer cell · CD56 · Activating ligand MICA $\cdot$ Benign breast disease

\section{Introduction}

The past two decades have seen a substantial expansion in the proposed role of the immune system in protecting against carcinogenesis, outlined in various models ranging from immunosurveillance $[1,2]$ to immunoediting $[3,4]$ and tumor escape [5-7]. Current literature supports a role specifically for natural killer (NK) cells in immune-mediated 
protection against breast cancer, with increased presence of NK cells associated with an increase in effective breast tumor surveillance [8-14].

In the immunosurveillance hypothesis, epithelial cells with early DNA damage and stress may be recognized and cleared by the immune system, with NK cells playing a central role [15]. CD56, also known as neural cell adhesion molecule (NCAM), is a glycoprotein expressed on the surface of NK cells. CD56+ NK cells have been suggested to play a bifunctional role in early immune response through both highly cytotoxic behavior and upregulation of proinflammatory cytokine release [16].

MHC class I polypeptide-related sequence A (MICA) is an innate ligand for NKG2D, an activating receptor found on NK cells and T cells [17]. MICA may be a marker for early cellular stress, as it has been shown to be upregulated in conditions of heat shock [18] and oxidative stress [19]. MICA is largely absent in normal epithelium, but present in many epithelial tumors [20]. Once activated by MICA via NKG2D, NK cells have the ability to kill self-cells deficient in MHC class I molecules, a property important to immunosurveillance as tumor cells are known to evade the adaptive immune system through MHC class I downregulation [15].

To our knowledge, the presence of NK cells and their activating ligands in benign and premalignant human breast tissue has not been explored. We wished to explore the immunosurveillance role of NK cells in premalignant breast tissues by quantitating NK cell infiltrates and an activating marker of early cellular stress, MICA, in benign biopsy tissues from women who did and did not develop subsequent breast cancer. In this study, we carried out and quantified CD56 and MICA immunostaining in human breast tissues to investigate if and how CD56+ NK cell and NK cell ligand densities vary in nonmalignant breast lobules (1) between women who develop breast cancer and women who remain cancer-free, (2) according to age, (3) according to lobular involution, a histologic risk factor for cancer [21], and (4) according to fibrocystic status/epithelial proliferation, another established risk factor [22, 23].

\section{Methods}

\section{Tissue samples}

Approval was obtained from the Institutional Review Board to conduct this research. The investigation was carried out in a nested case-control design derived from the Mayo Clinic Benign Breast Disease (BBD) Cohort, a prospectively maintained cohort of $>13,000$ women who underwent benign breast biopsy at Mayo Clinic between 1967 and 2001 [22]. Cases were women with BBD who subsequently developed breast cancer; controls had similar follow-up but did not develop breast cancer. Cases were matched to controls on age at biopsy, year of biopsy, and time to follow-up [24]. Fifty case-control pairs (100 tissue samples) were randomly selected for this intensive study. For each sample, serial tissue sections underwent the following stains: H\&E, CD56, and MICA. Six of the 50 pairs had an inadequate CD56- or MICA-stained section and were excluded, leaving 44 pairs (88 samples) in the final analysis group.

\section{Histologic review}

H\&E-stained sections were reviewed by the study pathologist. Each sample was characterized for two "global" impressions of the entire slide: overall histologic impression and lobular involution, both of which are strongly associated with breast cancer risk [21, 22]. Histologic impression is based upon the highest degree of epithelial abnormality present within the sample, and is categorized as nonproliferative disease, proliferative disease without atypia, or atypical hyperplasia [22]. Age-related lobular involution was categorized as none (1-24\% of normal lobules in tissue are involuted), partial (25-74\%), or complete ( $\geq 75 \%$ ) [21].

\section{Individual lobule annotation and histologic features}

For each tissue sample, up to ten representative lobules were selected by the pathologist for detailed study (Fig. 1a). Each selected lobule was assigned a tracking number on the H\&E stain, and the same lobules were annotated on the corresponding immunostained serial sections for quantification of CD56 and MICA staining. The pathologist characterized each individually numbered lobule on $\mathrm{H} \& \mathrm{E}$ stain as either normal or fibrocystic. Normal appearing lobules were further characterized individually as not involuted, partially involuted, or completely involuted. Fibrocystic lobules were sub-classified as nonproliferative, proliferative without atypia, or proliferative with atypia, according to the degree of epithelial proliferation. Photomicrographs of representative lobules at $\times 400$ magnification were obtained using an Olympus 400 camera attached to a microscope.

\section{Immunohistochemical staining}

Immunohistochemical staining procedures were performed as described previously [25]. CD56 immunostaining was performed with 1:25 dilution of DAKO M7304 Natural Killer Cell-NCAM, and MICA staining utilized 1:200 dilution of Abcam ab62540. 
Fig. 1 Histologic analysis of breast lobules. a H\&E stain on a biopsy section, with representative lobules identified by pathologist. b MICA staining and lobule annotation on a serial section from the same biopsy as (a). c Annotation for analysis of a lobule stained for MICA. d Computer analysis run for MICA staining on lobule in (c). e Annotation for analysis of a lobule stained for CD56. f Computer analysis run for CD56 staining on lobule in (e). Analyses identify low-intensity MICA and CD56 reporter, moderate intensity, and strong intensity, in addition to normal cells, using yellow, orange, red, and blue highlights, respectively. Moderate intensity staining thresholds were used for all analyses

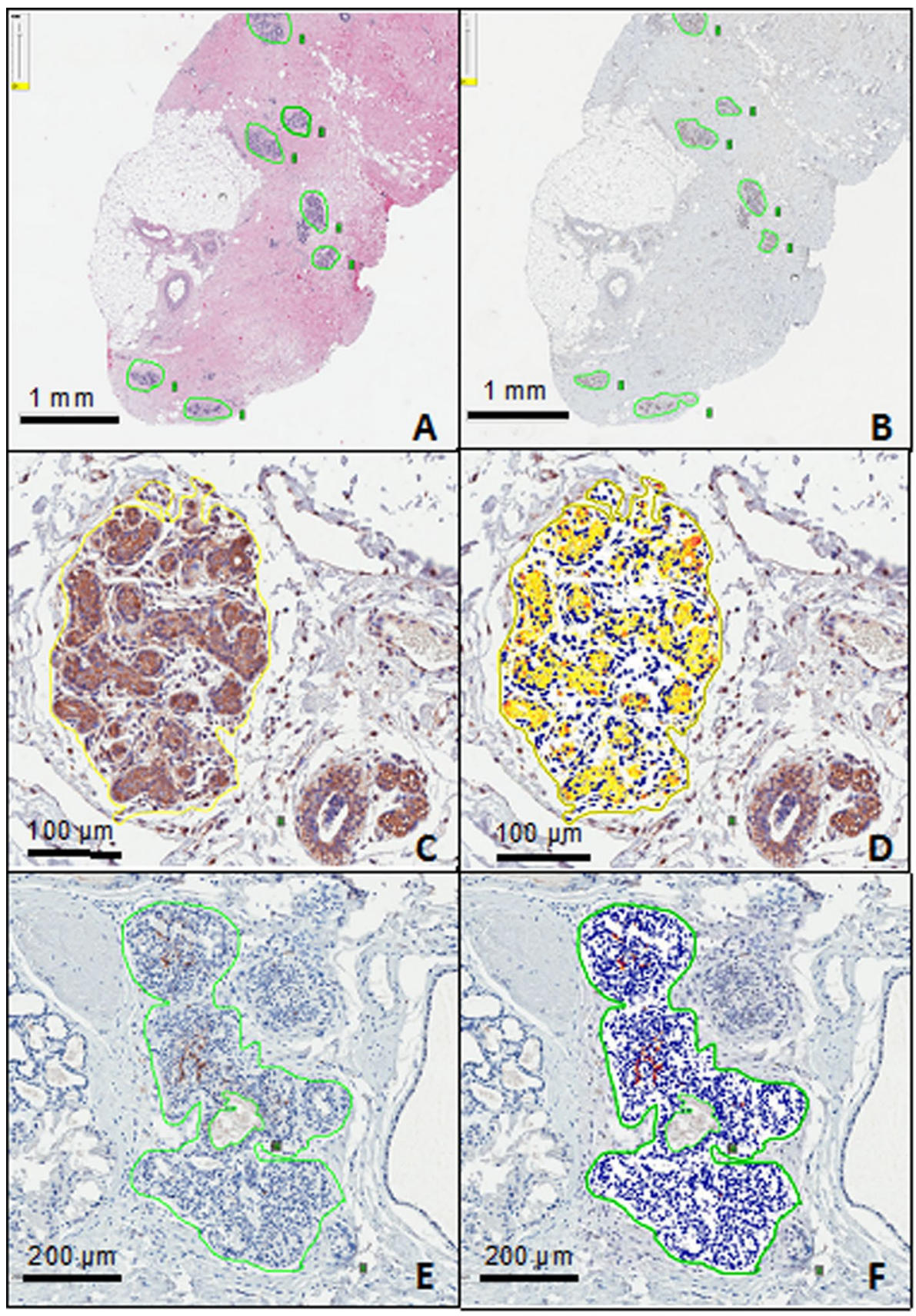

\section{Slide digitization and lobule annotation}

Slide digitization and lobule annotation procedures were described previously [26]. By the methods outlined, whole slide digital images of all 264 breast biopsy tissue sections (H\&E, anti-MICA, and anti-CD56 stains from each subject) were digitally scanned with the Aperio ScanScope AT2 slide scanner (Leica Biosystems, Buffalo Grove, IL) using the $20 \times$ objective lens. Boundaries of lobules were carefully marked for analysis and were considered to be the boundaries of the outermost epithelial cells in the lobule, with interlobular stroma and adipocytes excluded to the extent possible (Fig. 1b).

\section{Digital image analysis}

After lobule annotation, levels of positive staining for CD56 and MICA were quantified using digital imaging software (Aperio Technologies). The manufacturer's FDA-approved positive pixel count algorithms were used with optimization of the parameters for the CD56 and MICA stains [27]. The software uses specified threshold levels of pixel 
characteristics (Red Green Blue values and intensity levels) to identify and quantify positive staining levels and a digital color overlay to visually label pixels according to computed level of staining (Fig. 1c-f). The algorithm was applied uniformly to all annotated lobules across all samples. The algorithm calculated number of positively stained pixels and total number of pixels in each lobule analyzed. Levels of CD56 and MICA staining were calculated as a ratio of stained pixels to total pixels, in order to approximate the density of these markers throughout each lobule.

\section{Statistical analysis}

Analyses were performed at both the per-subject and perlobule level. To obtain per-subject estimates of MICA and CD56 percent positive pixel values, the medians within each subject were calculated across all lobules. Values for paired cases and controls were compared using Wilcoxon signedrank tests. Other per-subject analyses to explore associations of MICA and CD56 with patient-level variables including age, global degree of lobular involution, and global histologic impression were performed using Kruskal-Wallis tests for univariate analysis and general linear models for multivariate analysis. At the per-lobule level, the correlation between the lobule-specific MICA and CD56 percent positive pixels was examined with scatter plots and estimated using Spearman's rank correlation coefficient. Associations between lobule type and both MICA and CD56 values at the per-lobule level were assessed using linear mixed effects regression models with a random intercept for each subject to account for the correlation among multiple lobules from the same sample. Linear contrasts were used to test pairwise differences between lobule types within the mixed models. Due to a strong right skew, the Van der Waerden transformation was applied to MICA and CD56 percent values prior to modeling. The degree of within-sample variability across multiple lobules for MICA and CD56 was estimated by calculating a coefficient of variation (CV) for each sample as $100 \times$ (within-sample SD/within-sample mean). Analysis was performed using SAS Version 9.3. $p$ values $<0.05$ were considered statistically significant.

\section{Results}

\section{Characteristics of patients in analysis group}

The median age of study subjects was 52 (range 35-73) at the time of biopsy (cases and controls were matched on age). The majority of biopsies were excisional (72\%). Tissue samples exhibited a range of benign histologic findings [nonproliferative changes (36\%), proliferative changes without atypia (43\%), and atypical hyperplasia (21\%)] and degree of lobular involution [no involution (25\%), partial (41\%), and complete (34\%)]. These data are shown by case/ control status in Table 1. The median time from biopsy to
Table 1 Characteristics of patients with tissues included in BBD analysis presented by risk group

\begin{tabular}{llll}
\hline Variable & BBD case $(N=44)$ & BBD control $(N=44)$ & Total $(N=88)$ \\
\hline $\begin{array}{l}\text { Age at benign biopsy } \\
\text { Median (range) }\end{array}$ & $52(35-73)$ & $51.5(36-73)$ & $52(35-73)$ \\
Age category & & & \\
$\quad<45$ & $10(22.7 \%)$ & $10(22.7 \%)$ & $20(22.7 \%)$ \\
$45-55$ & $17(38.6 \%)$ & $16(36.4 \%)$ & $33(37.5 \%)$ \\
$>55$ & $17(38.6 \%)$ & $18(40.9 \%)$ & $35(39.8 \%)$ \\
Biopsy type & & & $3(3.4 \%)$ \\
Core biopsy followed by excisional biopsy & $1(2.3 \%)$ & $2(4.5 \%)$ & $22(25.0 \%)$ \\
Core biopsy only & $14(31.8 \%)$ & $8(18.2 \%)$ & $63(71.6 \%)$ \\
Excisional biopsy only & $29(65.9 \%)$ & $34(77.3 \%)$ & $32(36.4 \%)$ \\
Histologic impression & & & $38(43.2 \%)$ \\
Nonproliferative & $14(31.8 \%)$ & $18(40.9 \%)$ & $18(20.5 \%)$ \\
Proliferative disease without atypia & $19(43.2 \%)$ & $19(43.2 \%)$ & $3(15.9 \%)$ \\
Atypical hyperplasia & $11(25.0 \%)$ & $7(24.7 \%)$ \\
Lobular involution & & & $35(41.2 \%)$ \\
Not applicable* & 3 & 0 & $29(34.1 \%)$ \\
None & $12(29.3 \%)$ & $9(20.5 \%)$ & \\
Partial & $20(48.8 \%)$ & $15(34.1 \%)$ & $20(45.5 \%)$ \\
Complete & $9(22.0 \%)$ & & \\
\hline
\end{tabular}

*For three samples, there were no normal lobules and therefore global evaluation of involution could not be assessed 
cancer in cases was 8.3 years (range 1.4-16.2 years), and the median length of follow-up in controls was 16.2 years (range 7.3-23.1 years). The cancers occurring among the 44 cases included 13 DCIS and 31 invasive cancers.

\section{Characteristics of individual lobules}

Among the 88 tissue samples, 770 lobules were annotated and studied (382 lobules in cases and 388 in controls). Of the 770 lobules, $43 \%$ were normal and $57 \%$ were fibrocystic. Of the 438 fibrocystic lobules, about half (51\%) exhibited nonproliferative changes, $45 \%$ exhibited proliferative changes without atypia, and $4 \%$ exhibited atypical hyperplasia. Lobules demonstrated a range of involution, with $18 \%$ showing no involution, 39\% showing partial involution, and $43 \%$ showing complete involution. These data as well as a breakdown by case status are presented in Table 2.

\section{Per-subject analyses}

The first analyses performed were on the per-subject level in an attempt to uncover any associations between CD56+ cell infiltration, MICA staining, and features of age, global involution status, global histologic impression, and case-control status.

\section{Association of age and overall involution with MICA and CD56 levels}

Both MICA and CD56 showed a significant association ( $p=0.005$ and $p=0.04$ ) with age at the per-subject level although in opposite directions (Fig. 2): MICA was highest in younger women ( $<45$ years), while CD56 was highest in older women ( $>55$ years). MICA was not associated with global involution status, while CD56 was significantly higher for women with more complete involution $(p=0.02)$; however, this result represented confounding with age since CD56 was highest in women aged $>55$, who are also those most likely to have complete involution. In a multivariate analysis including age, involution, histologic impression, and case status at the per-subject level, only age remained significantly associated with MICA $(p=0.005)$ and CD56 $(p=0.03)$.

\section{Global histologic impression and MICA/CD56}

Both MICA and CD56 percent values were found to be highest in breast tissue samples exhibiting nonproliferative changes, with stepwise decreases in samples with proliferative epithelial changes without atypia and with atypical hyperplasia, but these differences were not significant ( $p=0.64$ and 0.85 for MICA and CD56, respectively). Median levels of MICA were $12.9 \%$ for nonproliferative changes, $9.9 \%$ for proliferative changes without atypia, and $9.2 \%$ for atypical hyperplasia; median values for CD56 for these groups were $0.64,0.51$, and $0.42 \%$, respectively.

\section{Case status and MICA/CD56}

Women who were BBD controls (no subsequent breast cancer) did not have statistically different overall tissue values of MICA nor CD56 as compared to BBD cases (patients that later developed cancer). Median MICA values were $10.0 \%$ in controls and $10.8 \%$ in cases $(p=0.70)$, whereas median CD56 values were $0.64 \%$ in controls and $0.39 \%$ in cases $(p=0.62)$.

\section{Per-lobule analyses}

We also performed analyses on a per-lobule basis in order to further evaluate associations of MICA and CD56 with histologic features that vary for each lobule within a
Table 2 Characteristics of lobules included in BBD analysis presented by risk group

\begin{tabular}{lccc}
\hline Variable & $\begin{array}{c}\text { BBD case } \\
(N=382)\end{array}$ & BBD control $(N=388)$ & Total $(N=770)$ \\
\hline $\begin{array}{l}\text { Lobule histologic impression } \\
\text { Normal }\end{array}$ & $140(36.6 \%)$ & $192(49.5 \%)$ & $332(43.1 \%)$ \\
Fibrocystic & $242(63.4 \%)$ & $196(50.5 \%)$ & $438(56.9 \%)$ \\
Proliferative status among fibrocystic lobules & & \\
Nonproliferative & $118(48.8 \%)$ & $106(54.1 \%)$ & $224(51.1 \%)$ \\
Proliferative & $113(46.7 \%)$ & $84(42.9 \%)$ & $197(45.0 \%)$ \\
Atypia & $11(4.5 \%)$ & $6(3.1 \%)$ & $17(3.9 \%)$ \\
Involution status among normal lobules & & $60(18.1 \%)$ \\
None & $24(17.1 \%)$ & $36(18.8 \%)$ & $129(38.9 \%)$ \\
Partial & $64(45.7 \%)$ & $65(33.9 \%)$ & $143(43.1 \%)$ \\
Complete & $52(37.1 \%)$ & $91(47.4 \%)$ & \\
\hline
\end{tabular}



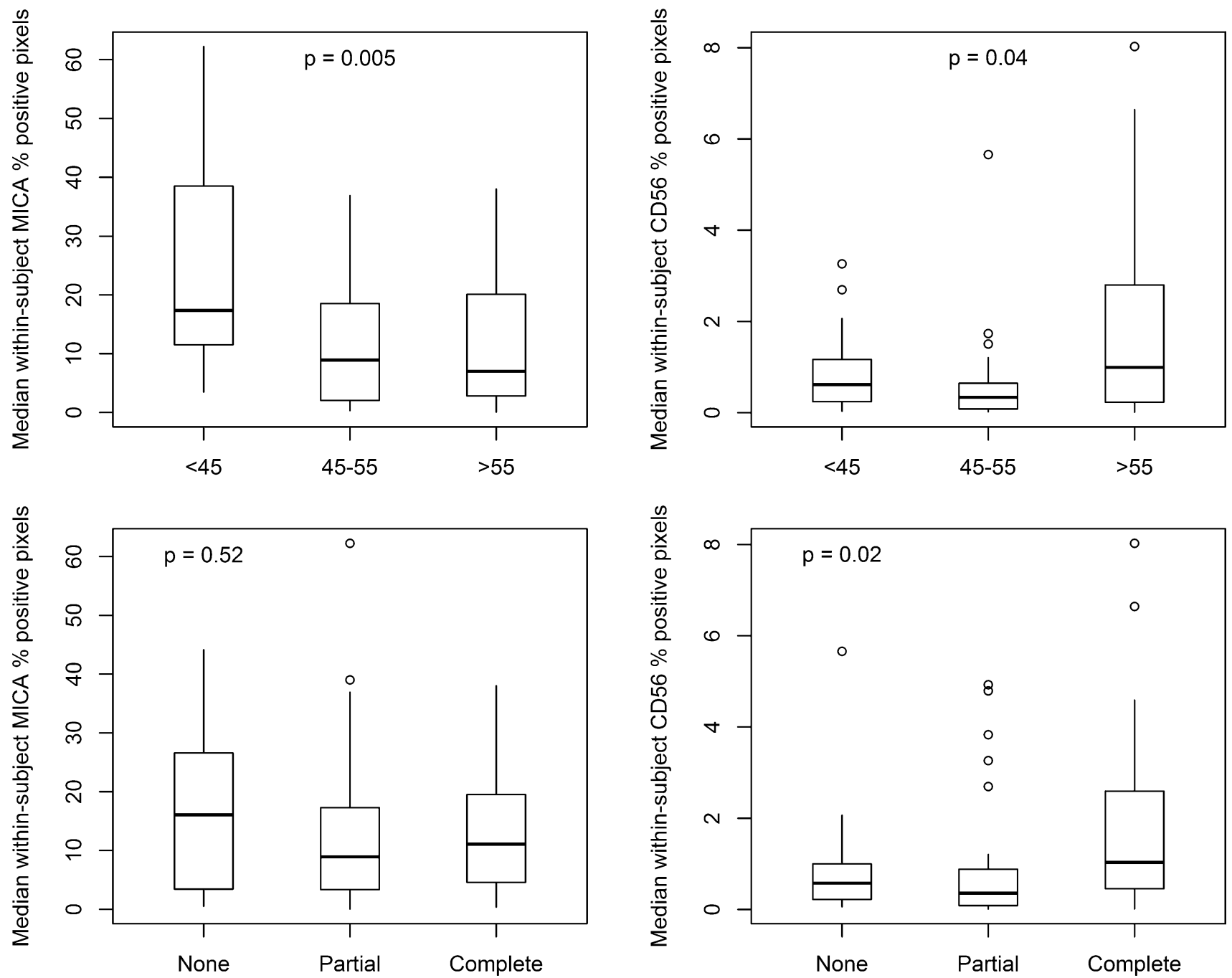

Fig. 2 Per-subject median values for MICA and CD56 positive pixel percentages by age group and global degree of involution. $p$ values are reported for univariate analyses. CD56 was significantly associated with both age and involution, while MICA was significantly

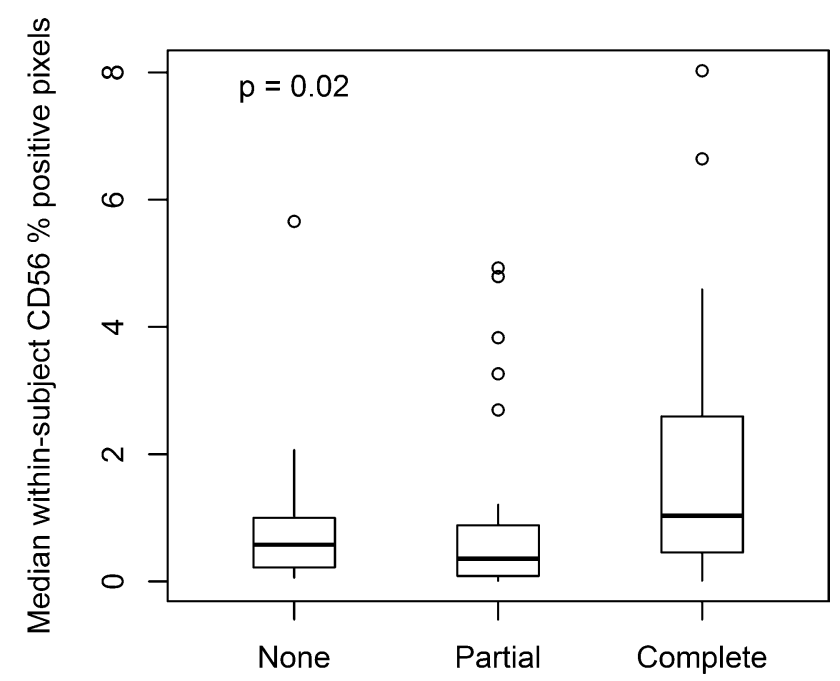

associated with age only. MICA and CD56 show nearly opposite trends with respect to age. Top and bottom of boxes in plot represent 75 th and 25 th percentile values, respectively

sample: fibrocystic status, degree of epithelial abnormality/proliferation, and involution status. Almost all lobules [98.1\% (755/770)] showed presence of both CD56+ cells and MICA, and substantial within-subject variability was observed across multiple lobules from a single sample (median $\mathrm{CV}=68 \%$ for MICA and median $114 \%$ for CD56, see Suppl Fig. 1 of MICA/CD56 plots on ten random samples). This variability across lobules provided additional rationale for performing per-lobule analyses.

\section{MICA and CD56 expression in fibrocystic versus normal lobules}

Compared to normal lobules, lobules that exhibited fibrocystic changes (with or without proliferation or atypia)

had significantly lower median levels of both MICA (7.0 compared to $12.2 \%, p<0.001)$ and CD56 (0.31 compared to $0.76 \%, p \leq 0.001)$. Pairwise comparisons showed that compared to normal lobules, CD56 staining was significantly lower in fibrocystic nonproliferative lobules $(p=0.005)$ and fibrocystic proliferative/atypical lobules $(p=0.003)$. The same trend was observed for MICA, with higher expression in normal lobules, with the difference being significant compared to fibrocystic proliferative/ atypia lobules $(p<0.001)$ but not significant compared to fibrocystic nonproliferative lobules ( $p=0.02$, Fig. 3 ). 


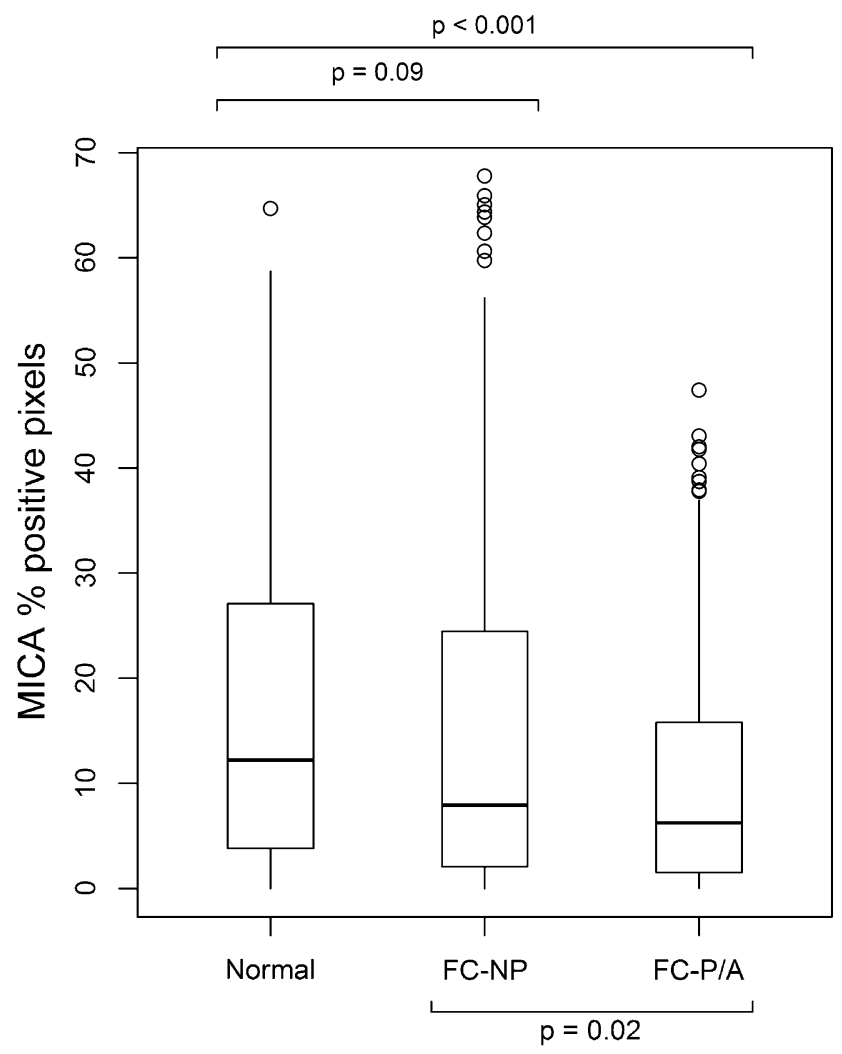

Fig. 3 Per-lobule median values for MICA and CD56 positive pixel percentages by fibrocystic status, pairwise comparisons. Fibrocystic lobules were further clustered by proliferative status (NP nonproliferative changes, $P / A$ proliferative changes without/or with atypia). $p$ values were adjusted for subject age and case status. Top and bot-

\section{MICA and CD56 expression in fibrocystic proliferative versus nonproliferative lobules}

When fibrocystic lobules were further classified by proliferative status into nonproliferative and proliferative/atypical, MICA showed lower levels in proliferative/atypical versus nonproliferative lobules (median $6.2 \%$ vs. $7.9 \%, p=0.02$ ), but CD56 did not (median $0.27 \%$ vs. $0.37 \%, p=0.80$, Fig. 3).

\section{MICA and CD56 by involution status in normal lobules}

Among normal lobules classified as exhibiting no, partial, or complete involution, MICA and CD56 percent values were higher for greater degrees of involution, but these differences were not significant ( $p=0.41$ and 0.36 for MICA and CD56, respectively).

\section{Correlation of MICA and CD56 levels}

At the per-lobule level, MICA and CD56 values showed a correlation of $r=0.28$ for all lobules combined. This

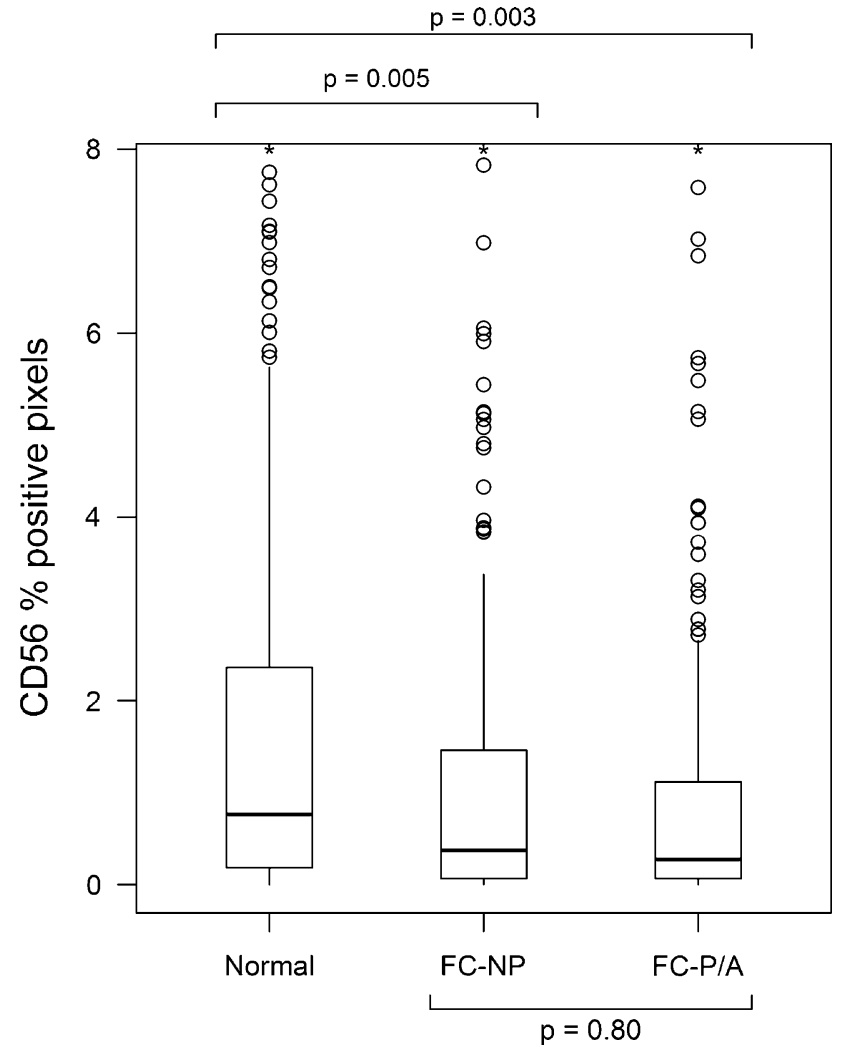

tom of boxes in plot represent 75 th and 25 th percentile values, respectively, and values beyond $8 \%$ pixels positive for CD56 were not included in the plot and were designated with asterisks. $p$ values were $<0.001$ for normal versus all fibrocystic lobules for both MICA and CD56

appears to be an age-dependent relationship, with only a weak correlation $(r<0.20)$ in those aged $<55$, but a moderate correlation of $r=0.46$ for women aged $>55$ (Suppl Fig. 2). In women aged $<45$, MICA seems high regardless of CD56 level, whereas for those aged $>55$ MICA remains higher in women with higher CD56 and is lower in women with lower CD56.

\section{Discussion}

MICA is a stress-inducible ligand that is expressed in many epithelial tumors [20]. It is an activating ligand for NKG2D, a receptor found on NK cells. When CD56+ NK cells are activated by MICA, they adopt a cytotoxic phenotype [17]. Through this mechanism, expression of MICA by premalignant cells in the absence of inhibitory signals promotes destruction by CD56 + NK cells before the mutated cells progress to cancer [28]. The current investigation quantitatively determined levels of CD56 and MICA expression in nonmalignant adult breast tissues with the hypothesis that lower levels of these markers would indicate suppressed NK 
cell immunoprotection in the breast epithelium and a higher incidence of cancer development.

In this study, both CD56 and MICA expressions were significantly lower in fibrocystic abnormal lobules compared to phenotypically normal lobules. Furthermore, MICA levels were lower in fibrocystic lobules with greater degree of abnormality. Higher CD56 levels and lower MICA levels were also each associated with older age, but had no association with involution status. Lastly, per-lobule MICA and CD56 expressions were positively correlated overall, with the strongest correlation in women over age 55 .

Breast tissues with increasing degrees of fibrocystic change and epithelial abnormality are associated with a higher risk of developing breast cancer [22]. Therefore, the finding that lobules with fibrocystic changes show significantly lower levels of CD56 and MICA than normal lobules is consistent with the premise that decreased levels of MICA-activated CD56+ NK cells contribute to an environment of easier immune escape. It is possible that premalignant epithelial changes in the tissue are in part genetically characterized by decreased release of NK cell chemotactic factors and decreased expression of NK cell-activating ligands, such as MICA. The correlation of lobular MICA and CD56 levels strengthens a hypothesis in which MICA and CD56+ NK cells function together within an anti-tumor cytotoxic system. However, given the lack of direct association between these markers and an eventual cancer diagnosis, further studies are needed to confirm a functional role of MICA and CD56+ NK cells in tumor immunosurveillance.

Given the well-established association between age and breast cancer risk [29], we expected both CD56 and MICA presence to decrease with age. Consistent with our expectation, MICA levels significantly decreased with age; however, we found that CD56 levels were significantly increased in women of older age. If MICA and CD56 play a role in breast cancer immunosurveillance, it is possible that in older women, immunosurveillance underperformance stems predominantly not from a decrease in the density of CD56+ NK cells, but a decrease in the cytotoxicity of CD56+ NK cells as a result of decreased activation by MICA. Though CD56 expression levels are highest in the oldest subject group, MICA expression is lowest in this group. In fact, MICA demonstrates a significant $(p=0.005)$ stepwise decrease in expression with increasing age, mirroring the trend observed in degree of histopathologic abnormality. CD56 does not show as consistent or significant $(p=0.04)$ a trend across age groups, and disappears upon controlling for other factors.

In considering these findings, one particular strength of the current study is the use of human breast tissue ex vivo, as very few prior studies have evaluated NK cells in benign human breast tissues. Another important strength of this study was its ability to analyze staining levels by individual lobules, which comprise a spectrum of histologic abnormalities. In evaluating the results, it became clear that subjectlevel analyses were too broad to usefully reflect the physiology of immunosurveillance, as each tissue is a collection of lobules with varying biology. Other strengths include scalability of method, quantitative data on MICA and CD56 densities, and evaluation of two different markers for NK cell activity.

The primary limitation of this study is that we cannot infer functional immune cell activity from the immunohistochemical stains. We did not differentiate between CD56 ${ }^{\mathrm{dim}}$ and CD56 $6^{\text {bright }}$ cells in this investigation, which have been reported to have different principal functions: $\mathrm{CD} 56^{\mathrm{dim}}$ cells appear to be primarily cytolytic in function, and CD56 ${ }^{\text {bright }}$ cells primarily produce cytokines [16]. A second limitation of the study is that we did not find a direct association between either CD56 or MICA with risk of breast cancer in the per-subject analyses. Further limitations of the study include a relatively small number of women and lack of consideration for other risk factors including family history, BMI, life habits, and menopausal status. Lastly, we recognize that there are several described activating and inhibitory receptors on NK cells for which we did not stain in this study, [30] in addition to a number of other immune cells implicated in breast tumor surveillance [24].

\section{Conclusion}

In summary, we found that CD56+ NK cells and MICA were present in benign breast tissues at significantly lower levels in lobules with epithelial abnormalities. Furthermore, CD56 expression was slightly positively correlated with age, while MICA expression was robustly negatively correlated with age. These findings could be explained by a hypothesis in which natural killer (NK) cells have an orchestrated cytotoxic functionality in the immunosurveillance of the normal breast that is compromised with age and in lobules with epithelial abnormalities. Specifically, these results could support a model in which proper MICA expression promotes CD56+ NK cell destruction of lobular cells that are cancerous or at risk for becoming cancerous. Further studies are necessary to refine the relationship between breast tissue histology and NK cell infiltration and to confirm a functional role of these cells in immunosurveillance.

Acknowledgements This research was supported by grants from Susan G. Komen for the Cure ${ }^{\circledR}$ and by the University of Notre Dame. Samples from the Susan G. Komen for the Cure ${ }^{\circledR}$ Tissue Bank at the IU Simon Cancer Center were used in this study. We thank contributors, including Indiana University, who collected samples used in this study, as well as donors and their families, whose help and participation made this work possible. Sincere thanks also to Alexander Pearlman for assistance with manuscript preparation. 


\section{Compliance with ethical standards}

Conflict of interest The authors have no conflict of interest.

Open Access This article is distributed under the terms of the Creative Commons Attribution 4.0 International License (http://creativecommons.org/licenses/by/4.0/), which permits unrestricted use, distribution, and reproduction in any medium, provided you give appropriate credit to the original author(s) and the source, provide a link to the Creative Commons license, and indicate if changes were made.

\section{References}

1. Klein G (1976) Immune surveillance- a powerful mechanism with a limited range. Natl Cancer Inst Monogr 44:109-113

2. Thomas L (1982) On immunosurveillance in human cancer. Yale J Biol Med 55(3-4):329-333

3. Dunn GP, Bruce AT, Ikeda H, Old LJ, Schreiber RD (2002) Cancer immunoediting: from immunosurveillance to tumor escape. Nat Immunol 3(11):991-998

4. Kim R, Emi M, Tanabe K (2007) Cancer immunoediting from immune surveillance to immune escape. Immunology 121(1):114. https://doi.org/10.1111/j.1365-2567.2007.02587.x

5. Igney FH, Krammer PH (2002) Immune escape of tumors: apoptosis resistance and tumor counterattack. J Leukocyte Biol 71(6):907-920

6. Bogen B (1996) Peripheral T cell tolerance as a tumor escape mechanism: deletion of CD4+ $\mathrm{T}$ cells specific for monoclonal immunoglobulin idiotype secreted by a plasmacytoma. Euro J Immunol 26(11):2671-2679. https://doi.org/10.1002/ eji.1830261119

7. Maeurer MJ, Gollin SM, Martin D, Swaney W, Bryant J, Casatelli C, Robbins P, Parmiani G, Storkus WJ, Lotze MT (1996) Tumor escape from immune recognition: lethal recurrent melanoma in a patient associated with downregulation of the peptide transporter protein TAP-1 and loss of expression of immunodominant MART-1/Melan-A antigen. J Clin Invest 98(7):1633-1641. https://doi.org/10.1172/JCI118958

8. Kajitani K, Tanaka Y, Arihiro K, Kataoka T, Ohdan H (2012) Mechanistic analysis of the antitumor efficacy of human natural killer cells against breast cancer cells. Breast Cancer Res Treat 134(1):139-155. https://doi.org/10.1007/s10549-011-1944-x

9. Dewan MZ, Terunuma H, Takada M, Tanaka Y, Abe H, Sata T, Toi M, Yamamoto N (2007) Role of natural killer cells in hormone-independent rapid tumor formation and spontaneous metastasis of breast cancer cells in vivo. Breast Cancer Res Treat 104(3):267-275. https://doi.org/10.1007/ s10549-006-9416-4

10. Diessner J, Bruttel V, Becker K, Pawlik M, Stein R, Häusler S, Dietl J, Wischhusen J, Hönig A (2013) Targeting breast cancer stem cells with HER2-specific antibodies and natural killer cells. Am J Cancer Res 3(2):211-220

11. Divino CM, Chen SH, Yang W, Thung S, Brower ST, Woo SL (2000) Anti-tumor immunity induced interleukin-12 gene therapy in a metastatic model of breast cancer is mediated by natural kills cells. Breast Cancer Res Treat 60(2):129-134

12. Roberti MP, Mordoh J, Levy EM (2012) Biological role of NK cells and immunotherapeutic approaches in breast cancer. Front Immunol. https://doi.org/10.3389/fimmu.2012.00375

13. Jiang X, Ellison SJ, Alarid ET, Shapiro DJ (2007) Interplay between the levels of estrogen and estrogen receptor controls the level of the granzyme inhibitor, proteinase inhibitor
9 and susceptibility to immune surveillance by natural killer cells. Oncogene 26(28):4106-4114. https://doi.org/10.1038/ sj.onc. 1210197

14. Croci S, Nanni P, Palladini A, Nicoletti G, Grosso V, Benegiamo G, Landuzzi L, Lamolinara A, Ianzano ML, Ranieri D, Dall'Ora M, Iezzi M, De Giovanni C, Lollini PL (2015) Interleukin-15 is required for immunosurveillance and immunoprevention of HER2/neu-driven mammary carcinogenesis. Breast Cancer Res 17:70. https://doi.org/10.1186/s13058-015-0588-x

15. Watzl C, Long EO (2000) Exposing tumor cells to killer cell attack. Nat Med 6(8):867-868. https://doi.org/10.1038/78624

16. Poli A, Michel T, Thérésine M, Andrès E, Hentges F, Zimmer J (2009) CD56bright natural killer (NK) cells: an important NK cell subset. Immunology 126(4):458-465. https://doi. org/10.1111/j.1365-2567.2008.03027.x

17. Bauer S, Groh V, Wu J, Steinle A, Phillips JH, Lanier LL, Spies T (1999) Activation of NK cells and T cells by NKG2D, a receptor for stress-inducible MICA. Science 285(5428):727729. https://doi.org/10.1126/science.285.5428.727

18. Groh VS, Bahram S, Bauer S, Herman A, Beauchamp M, Spies T (1996) Cell stress-regulated human major histocompatibility complex class I gene expressed in gastrointestinal epithelium. Proc Natl Acad Sci U S A 93(22):12445-12450

19. Yamamoto K, Fujiyama Y, Andoh A, Bamba T, Okabe H (2001) Oxidative stress increases MICA and MICB gene expression in the human colon carcinoma cell line $(\mathrm{CaCo}-2)$. Biochim Biophys Acta 1526(1):10-12

20. Groh V, Rhinehart R, Secrist H, Bauer S, Grabstein KH, Spies $\mathrm{T}$ (1999) Broad tumor-associated expression and recognition by tumor-derived $\Gamma \delta$ T cells of MICA and MICB. Proc Natl Acad Sci 96(12):6879-6884. https://doi.org/10.1073/pnas.96.12.6879

21. Milanese TR, Hartmann LC, Sells TA, Frost MH, Vierkant RA, Maloney SD, Pankratz VS, Degnim AC, Vachon CM, Reynolds CA, Thompson RA, Melton LJ 3rd, Goode EL, Visscher DW (2006) Age-related lobular involution and risk of breast cancer. J Natl Cancer Inst 98(22):1600-1607. https://doi.org/10.1093/ jnci/djj439

22. Hartmann LC, Sellers TA, Frost MH, Lingle WL, Degnim AC, Ghosh G, Vierkant RA, Maloney SD, Pankratz VS, Hillman DW, Suman VJ, Johnson J, Blake C, Tlsty T, Vachon CM, Melton LJ 3rd, Visscher DW (2005) Benign breast disease and the risk of breast cancer. N Engl J Med 353(3):229-237. https://doi. org/10.1056/NEJMoa044383

23. Hartmann LC, Radisky DC, Frost MH, Santen RJ, Vierkant RA, Benetti LL, Tarabishy Y, Ghosh K, Visscher DW, Degnim AC (2014) Understanding the premalignant potential of atypical hyperplasia through its natural history: a longitudinal cohort study. Cancer Prev Res (Phila) 7(2):211-217

24. Degnim AC, HoskiN TL, Arshad M, Frost MH, Winham SJ, Brahmbhatt RA, Pena A, Carter JM, Stallings-Mann M, Murphy L, Denison L, Vachon CM, Knutson KL, Radisky DC, Visscher DW (2017) Alterations in the immune cell composition in premalignant breast tissue that precede breast cancer development. Clin Cancer Res. https://doi.org/10.1158/1078-0432. CCR-16-2026

25. Radisky DC, Santisteban M, Berman HK, Gauthier ML, Frost MH, Reynolds CA, Vierkant RA, Pankratz VS, Visscher DW, Tlsty TD, Hartmann LC (2011) p16(INK4a) expression and breast cancer risk in women with atypical hyperplasia. Cancer Prev Res 4(12):1953-1960. https://doi.org/10.1158/1940-6207. CAPR-11-0282

26. Degnim AC, Brahmbhatt RD, Radisky DC, Hoskin TL, Stallings-MannM Laudenschlager M, Mansfield A, Frost MH, Murphy L, Knutson K, Visscher DW (2014) Immune cell quantitation in normal breast tissue lobules with and without 
lobulitis. Breast Cancer Res Treat 144(3):539-549. https://doi. org/10.1007/s10549-014-2896-8

27. Positive Pixel Count Algorithm User's Guide (2015) Aperio Technologies, Inc. http://tmalab.jhmi.edu/aperiou/userguides/ Positive_Pixel.pdf. Accessed 3 July 2015

28. Pende D, Rivera P, Marcenaro S, Chang CC, Biassoni R, Conte R, Kubin Cosman D, Ferrone S, Moretta L, Moretta A (2002) Major histocompatibility complex class I-related chain A and UL16-binding protein expression on tumor cell lines of different histotypes: analysis of tumor susceptibility to
NKG2D-dependent natural killer cell cytotoxicity. Cancer Res 62(21):6178-6186

29. McPherson K, Steel CM, Dixon J (2000) Breast cancerepidemiology, risk factors, and genetics. BMJ Br Med J 321(7261):624-628

30. Farnault L, Sanchez C, Baier C, Le Treut T, Costello RT (2012) Hematological malignancies escape from NK cell innate immune surveillance: mechanisms and therapeutic implications. J Immunol Res 2012:e421702. https://doi.org/10.1155/2012/421702 